\title{
On a space of entire functions rapidly decreasing on the real axis and its Fourier transform
}

\author{
Il'dar Kh. Musin'1 • Marat I. Musin'²
}

Received: 22 August 2014 / Revised: 27 February 2015 / Accepted: 1 March 2015 /

Published online: 3 April 2015

(C) Springer International Publishing AG 2015

\begin{abstract}
A weighted space of entire functions rapidly decreasing on the real axis is considered. The growth of these functions along the imaginary axis is controlled by some system of weight functions. The Fourier transforms of functions of this space are described as some space of infinitely differentiable functions on $\mathbb{R}$. An equivalent description of the considered space in terms of estimates on derivatives of functions on the real axis is obtained.
\end{abstract}

Keywords Gelfand-Shilov spaces · Fourier transform · Entire functions · Convex functions

Mathematics Subject Classification $32 \mathrm{~A} 15 \cdot 46 \mathrm{E} 10 \cdot 46 \mathrm{~F} 05 \cdot 42 \mathrm{~A} 38$

The research of the first author was supported by Grants from RFBR (14-01-00720, 15-01-01661).

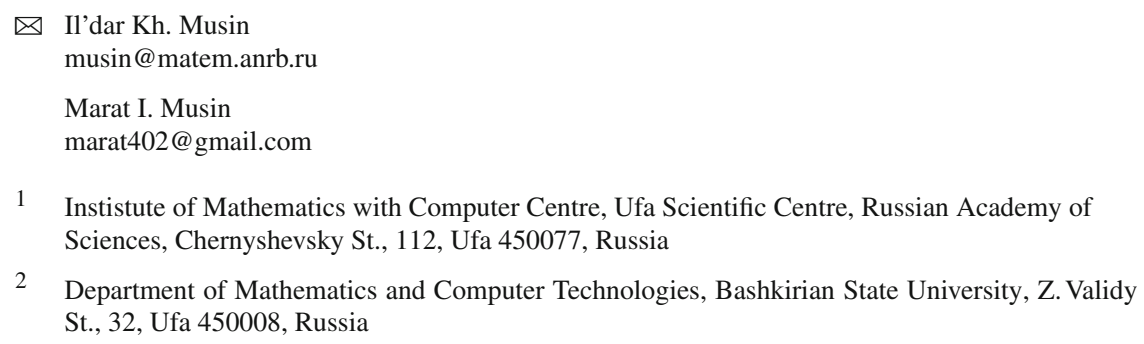




\section{Introduction}

\subsection{On the problem}

Let $\Phi=\left\{\varphi_{m}\right\}_{m=1}^{\infty}$ be a family of nondecreasing continuous functions $\varphi_{m}:[0, \infty) \rightarrow$ $\mathbb{R}$ such that for each $m \in \mathbb{N}$ :

(i $)_{1} \lim _{x \rightarrow+\infty} \varphi_{m}(x) / x=+\infty$;

(i $\left.i_{2}\right)$ for each $A>0$ there exists a constant $C(m, A)>0$ such that

$$
\varphi_{m}(x)+A \ln (1+x) \leq \varphi_{m+1}(x)+C(m, A), \quad x \geq 0 .
$$

Moreover, in Theorems 1.2-1.4 the following additional conditions on $\Phi$ will be imposed (separately or together):

(i3) for each $k \in \mathbb{N}$ there exist constants $\sigma_{k}>1$ and $\gamma_{k}>0$ such that

$$
\varphi_{k}\left(\sigma_{k} x\right) \leq \varphi_{k+1}(x)+\gamma_{k}, \quad x \geq 0
$$

$\left(\mathrm{i}_{4}\right)$ for each $k \in \mathbb{N}$ there exist numbers $\lambda_{k}>1, h_{k} \in\left(1, \sigma_{k+1}\right)$ and $l_{k}>0$ such that

$$
\lambda_{k} \varphi_{k}(x) \leq \varphi_{k+1}\left(h_{k} x\right)+l_{k}, \quad x \geq 0 .
$$

Let $H(\mathbb{C})$ be a space of entire functions on $\mathbb{C}$. For each $v \in \mathbb{N}$ and $k \in \mathbb{Z}_{+}$define the space

$$
E_{k}\left(\varphi_{\nu}\right)=\left\{f \in H(\mathbb{C}): p_{v, k}(f)=\sup _{z \in \mathbb{C}} \frac{|f(z)|(1+|z|)^{k}}{e^{\varphi_{v}(|\operatorname{Im} z|)}}<\infty\right\} .
$$

Let $E\left(\varphi_{\nu}\right)=\bigcap_{k=0}^{\infty} E_{k}\left(\varphi_{\nu}\right), E(\Phi)=\bigcup_{\nu=1}^{\infty} E\left(\varphi_{\nu}\right)$. Since $p_{\nu, k}(f) \leq p_{\nu, k+1}(f)$ for $f \in E_{k+1}\left(\varphi_{\nu}\right), E_{k+1}\left(\varphi_{\nu}\right)$ is continuously embedded in $E_{k}\left(\varphi_{\nu}\right)$. Endow $E\left(\varphi_{\nu}\right)$ with a projective limit topology of spaces $E_{k}\left(\varphi_{\nu}\right)$. Note that if $f \in E\left(\varphi_{v}\right)$ then $p_{\nu+1, k}(f) \leq e^{C(\nu, 1)} p_{\nu, k}(f)$ for each $k \in \mathbb{Z}_{+}$. This means that $E\left(\varphi_{\nu}\right)$ is continuously embedded in $E\left(\varphi_{v+1}\right)$ for each $v \in \mathbb{N}$. Supply $E(\Phi)$ with a topology of an inductive limit of spaces $E\left(\varphi_{\nu}\right)$.

Note that if $\Omega$ is a differentiable function on $[0, \infty)$ such that $\Omega(0)=\Omega^{\prime}(0)=0$ and its derivative is continuous, increasing and tending to infinity and functions $\varphi_{m}$ are defined on $[0, \infty)$ by the formula $\varphi_{m}(x)=\Omega(m x), m \in \mathbb{N}$, then $E(\Phi)$ is the Gelfand-Shilov space of $W^{\Omega}$-type. Recall that $W^{\Omega}$-type (as other $W$-type) spaces have been introduced in 1950s by Gurevich [2,3], and Gelfand and Shilov [1]. They described them by means of the Fourier transform and then applied this description to study the uniqueness of the Cauchy problem of partial differential equations.

In this paper we describe the space $E(\Phi)$ in terms of estimates on derivatives of functions on the real axis and study Fourier transform of functions of $E(\Phi)$ under additional conditions on $\Phi$. We would like to note the works of Sedletskii $[8,9]$ as closely related to the theme of our paper. 
Results of the paper could be useful for theory of differential and pseudo-differential operators, mathematical physics, time-frequency analysis and some other fields of analysis.

\subsection{Notation}

Denote by $\mathcal{B}$ the set of all functions $g \in C[0, \infty)$ such that $\lim _{x \rightarrow+\infty} g(x) / x=+\infty$. Let

$$
\begin{aligned}
V= & \{h \in \mathcal{B}: h \text { is convex on }[0, \infty)\}, \\
\mathcal{V}=\{h \in V: & h \text { is increasing on }[0, \infty) \text { with } h(0)=0\}, \\
V_{g}=\{h \in \mathcal{V}: & h \text { coincides with } g \text { on }\left[d_{h}, \infty\right), \text { where } d_{h} \\
& \text { is some positive number depending on } h\}, \quad g \in V .
\end{aligned}
$$

For $g \in \mathcal{B}$ let $g^{*}$ be the Young conjugate: $g^{*}(x)=\sup _{y \geq 0}(x y-g(y)), x \geq 0$. For a function $u:[0, \infty) \rightarrow \mathbb{R}$ let $u[e](x)=u\left(e^{x}\right), x \geq 0$. For brevity, functions $\varphi_{m}[e]$ are denoted by $\psi_{m}$.

The Fourier transform $\widehat{f}$ of $f \in E(\Phi)$ is defined by the formula

$$
\widehat{f}(x)=\int_{\mathbb{R}} f(\xi) e^{-i x \xi} d \xi, \quad x \in \mathbb{R} .
$$

\subsection{Main results}

Let $\Psi^{*}=\left\{\psi_{\nu}^{*}\right\}_{\nu=1}^{\infty}$. For each $v \in \mathbb{N}$ and $m \in \mathbb{Z}_{+}$let

$$
\mathcal{E}_{m}\left(\psi_{v}^{*}\right)=\left\{f \in C^{\infty}(\mathbb{R}): \mathcal{R}_{m, v}(f)=\sup _{\substack{x \in \mathbb{R} \\ n \in \mathbb{Z}_{+}}} \frac{(1+|x|)^{m}\left|f^{(n)}(x)\right|}{n ! e^{-\psi_{v}^{*}(n)}}<\infty\right\} .
$$

Let $\mathcal{E}\left(\psi_{v}^{*}\right)=\bigcap_{m=0}^{\infty} \mathcal{E}_{m}\left(\psi_{v}^{*}\right), \mathcal{E}\left(\Psi^{*}\right)=\bigcup_{\nu=1}^{\infty} \mathcal{E}\left(\psi_{v}^{*}\right)$.

The first two theorems, proved in Sect. 3, are aimed to describe functions of the space $E(\Phi)$ in terms of estimates of their derivatives on $\mathbb{R}$.

Theorem 1.1 Let $f \in E(\Phi)$. Then $f_{\mid \mathbb{R}} \in \mathcal{E}\left(\Psi^{*}\right)$.

Theorem 1.2 Let the family $\Phi$ satisfy condition ( $\left.\mathrm{i}_{3}\right)$. Then each function $f \in \mathcal{E}\left(\Psi^{*}\right)$ admits (a unique) extension to an entire function belonging to $E(\Phi)$.

The proofs of these theorems follow the known schemes from [1]. Also they allow to obtain an additional information on the structure of the space $E(\Phi)$. Namely, let

$$
\mathcal{H}_{k}\left(\varphi_{\nu}\right)=\left\{f \in H(\mathbb{C}): \mathcal{N}_{\nu, k}(f)=\sup _{z \in \mathbb{C}} \frac{|f(z)|(1+|z|)^{k}}{e^{\left(\psi_{v}^{*}\right)^{*}(\ln (1+|\operatorname{Im} z|))}}<\infty\right\},
$$


$v \in \mathbb{N}, k \in \mathbb{Z}_{+}, \mathcal{H}\left(\varphi_{\nu}\right)$ be a projective limit of spaces $\mathcal{H}_{k}\left(\varphi_{\nu}\right), \mathcal{H}(\Phi)$ be an inductive limit of spaces $\mathcal{H}\left(\varphi_{\nu}\right)$. In Sect. 3 we show that if the family $\Phi$ satisfies condition (i $\left.i_{3}\right)$ then $E(\Phi)=\mathcal{H}(\Phi)$, see Proposition 3.1.

Section 4 is devoted to characterization of the space $E(\Phi)$ by means of the Fourier transform under additional conditions on $\Phi$. For each $v \in \mathbb{N}$ and $m \in \mathbb{Z}_{+}$we define the normed space

$$
G_{m}\left(\psi_{v}^{*}\right)=\left\{f \in C^{m}(\mathbb{R}):\|f\|_{m, \psi_{v}^{*}}=\sup _{\substack{x \in \mathbb{R}, k \in \mathbb{Z}_{+} \\ 0 \leq n \leq m}} \frac{\left|x^{k} f^{(n)}(x)\right|}{k ! e^{-\psi_{v}^{*}(k)}}<\infty\right\} .
$$

Let $G\left(\psi_{v}^{*}\right)=\bigcap_{m=0}^{\infty} G_{m}\left(\psi_{v}^{*}\right), G\left(\Psi^{*}\right)=\bigcup_{\nu=1}^{\infty} G\left(\psi_{v}^{*}\right)$. Since for each $v \in \mathbb{N}$ and $m \in \mathbb{Z}_{+}$,

$$
\|f\|_{m, \psi_{v}^{*}} \leq\|f\|_{m+1, \psi_{v}^{*},} \quad f \in G_{m+1}\left(\psi_{v}^{*}\right),
$$

$G_{m+1}\left(\psi_{v}^{*}\right)$ is continuously embedded in $G_{m}\left(\psi_{v}^{*}\right)$. Endow $G\left(\psi_{v}^{*}\right)$ with a topology defined by the family of norms $\|\cdot\|_{m, \psi_{v}^{*}}, m \in \mathbb{Z}_{+}$. Also note that for each $v \in \mathbb{N}$ and $m \in \mathbb{Z}_{+}$,

$$
\|f\|_{m, \psi_{v+1}^{*}} \leq e^{C(v, 1)}\|f\|_{m, \psi_{v}^{*}}, \quad f \in G_{m}\left(\psi_{v}^{*}\right)
$$

Hence, $G\left(\psi_{v}^{*}\right)$ is continuously embedded in $G\left(\psi_{v+1}^{*}\right)$. Supply $G\left(\Psi^{*}\right)$ with an inductive limit topology of spaces $G\left(\psi_{v}^{*}\right)$.

Theorem 1.3 Let $\Phi$ satisfy conditions $\left(\mathrm{i}_{3}\right)$ and $\left(\mathrm{i}_{4}\right)$. Then the Fourier transform $\mathcal{F}: E(\Phi) \ni f \rightarrow \widehat{f}$ establishes an isomorphism of spaces $E(\Phi)$ and $G\left(\Psi^{*}\right)$.

Further, let $\Phi^{*}=\left\{\varphi_{\nu}^{*}\right\}_{\nu=1}^{\infty}$. For each $v \in \mathbb{N}$ and $m \in \mathbb{Z}_{+}$define the normed space

$$
G S_{m}\left(\varphi_{v}^{*}\right)=\left\{f \in C^{m}\left(\mathbb{R}^{n}\right): q_{m, v}(f)=\sup _{\substack{x \in \mathbb{R} \\ 0 \leq n \leq m}} \frac{\left|f^{(n)}(x)\right|}{e^{-\varphi_{v}^{*}(|x|)}}<\infty\right\}
$$

For each $v \in \mathbb{N}$ let $G S\left(\varphi_{v}^{*}\right)=\bigcap_{m \in \mathbb{Z}_{+}} G S_{m}\left(\varphi_{v}^{*}\right)$. Let $G S\left(\Phi^{*}\right)=\bigcup_{v \in \mathbb{N}} G S\left(\varphi_{v}^{*}\right)$. Note that for each $v \in \mathbb{N}$ and $m \in \mathbb{Z}_{+}$,

$$
q_{m, v}(f) \leq q_{m+1, v}(f), \quad f \in G S_{m+1}\left(\varphi_{v}^{*}\right) .
$$

Hence, $G S_{m+1}\left(\varphi_{\nu}^{*}\right)$ is continuously embedded in $G S_{m}\left(\varphi_{v}^{*}\right)$. Endow $G S\left(\varphi_{v}^{*}\right)$ with a topology defined by the family of norms $q_{v, m}, m \in \mathbb{Z}_{+}$. Also for each $v \in \mathbb{N}$ and $m \in \mathbb{Z}_{+}$,

$$
q_{m, v+1}(f) \leq e^{C(v, 1)} q_{m, v}(f), \quad f \in G S_{m}\left(\varphi_{\nu}^{*}\right)
$$

Hence, $G S\left(\varphi_{v}^{*}\right)$ is continuously embedded in $G S\left(\varphi_{v+1}^{*}\right)$. Supply $G S\left(\Phi^{*}\right)$ with an inductive limit topology of spaces $G S\left(\varphi_{v}^{*}\right)$.

The main result of Sect. 5 is the following theorem. 
Theorem 1.4 Let functions of the family $\Phi$ be convex on $[0, \infty)$ and $\Phi$ satisfy condition $\left(\mathrm{i}_{3}\right)$. Then $G\left(\Psi^{*}\right)=G S\left(\Phi^{*}\right)$.

Note that if functions $\varphi_{m}$ are defined on $[0, \infty)$ by the formula $\varphi_{m}(x)=\Omega\left(\alpha_{m} x\right)$, where $\Omega$ is a differentiable function on $[0, \infty)$ such that $\Omega(0)=\Omega^{\prime}(0)=0$ and its derivative is continuous, increasing and tending to infinity and numbers $\alpha_{m}$ are positive and $\alpha_{m}<\alpha_{m+1}, m \in \mathbb{N}$, then the family $\Phi$ satisfies assumptions of Theorem 1.4.

\section{Auxiliary results}

The following two lemmas are essentially used in the next sections.

Lemma 2.1 Let $g \in \mathcal{B}$, then for each $M>0$ there exists a constant $A>0$ such that

$$
(g[e])^{*}(x) \leq x \ln \frac{x}{M}-x+A, \quad x>0 .
$$

Proof By assumption, for each $M>0$ we can find a number $A>0$ such that $g[e](y) \geq M e^{y}-A$ for all $y \geq 0$. Hence, for $x>0$,

$$
\begin{aligned}
(g[e])^{*}(x) & =\sup _{y>0}(x y-g[e](y)) \leq \sup _{y>0}\left(x y-M e^{y}\right)+A \\
& \leq \sup _{y \in \mathbb{R}}\left(x y-M e^{y}\right)+A=x \ln \frac{x}{M}-x+A .
\end{aligned}
$$

Corollary 2.1 Let $g \in \mathcal{B}$, then for each $b>0$ the series $\sum_{j=0}^{\infty} e^{(g[e])^{*}(j)} /\left(b^{j} j !\right)$ converges.

Lemma 2.2 Let $u, v \in \mathcal{B}$ and for some $\rho>0, \mu \geq 0$ and $C>0$,

$$
\rho u(x) \leq v(x+\mu)+C, \quad x \geq 0 .
$$

Then for all nonnegative numbers $\alpha$ and $\beta$ satisfying the equality $\alpha+\beta=\rho$ we have

$$
v^{*}(\alpha x+\beta y) \leq \alpha u^{*}(x)+\beta u^{*}(y)+\mu(\alpha x+\beta y)+A, \quad x, y \geq 0,
$$

where $A=\max \left(C, \rho \min _{\xi \geq 0} u(\xi)-\min _{\xi \geq 0} v(\xi)\right)$.

Proof For all $x, y, t \in[0, \infty)$ and nonnegative numbers $\alpha$ and $\beta$ satisfying the equality $\alpha+\beta=\rho$ we have

$$
\alpha u^{*}(x)+\beta u^{*}(y) \geq(\alpha x+\beta y) t-\rho u(t) \geq(\alpha x+\beta y) t-v(t+\mu)-C .
$$

Hence,

$$
\alpha u^{*}(x)+\beta u^{*}(y) \geq \sup _{\xi \geq \mu}((\alpha x+\beta y) \xi-v(\xi))-\mu(\alpha x+\beta y)-C .
$$


Note that for all $x, y \in[0, \infty)$,

$$
\begin{aligned}
& \sup _{\xi \in[0, \mu]}((\alpha x+\beta y) \xi-v(\xi)) \leq(\alpha x+\beta y) \mu-\min _{\xi \in[0, \mu]} v(\xi) \\
& \quad \leq(\alpha x+\beta y) \mu+\alpha u^{*}(x)+\beta u^{*}(y)+\rho \min _{\xi \geq 0} u(\xi)-\min _{\xi \geq 0} v(\xi)
\end{aligned}
$$

From this and inequality (1) we obtain that for all $x, y \geq 0$,

$$
v^{*}(\alpha x+\beta y) \leq \alpha u^{*}(x)+\beta u^{*}(y)+(\alpha x+\beta y) \mu+A,
$$

where $A=\max \left(C, \rho \min _{\xi \geq 0} u(\xi)-\min _{\xi \geq 0} v(\xi)\right)$.

\section{Alternative approach to description of $E(\Phi)$}

3.1 Proof of Theorem 1.1 Let $f \in E(\Phi)$, then $f \in E\left(\varphi_{\nu}\right)$ for some $v \in \mathbb{N}$. Fix $m \in \mathbb{Z}_{+}$. For all $x \in \mathbb{R}, n \in \mathbb{Z}_{+}$and $R>0$ we have

$$
(1+|x|)^{m} f^{(n)}(x)=\frac{n !}{2 \pi i} \int_{L_{R}(x)} \frac{(1+|x|)^{m} f(\zeta)}{(\zeta-x)^{n+1}} d \zeta
$$

where $L_{R}(x)=\{\zeta \in \mathbb{C}:|\zeta-x|=R\}$. From this we have

$$
\begin{aligned}
(1+|x|)^{m}\left|f^{(n)}(x)\right| & \leq n ! \max _{\zeta \in L_{R}(x)} \frac{(1+|\zeta-x|)^{m}(1+|\zeta|)^{m}|f(\zeta)|}{R^{n}} \\
& \leq \frac{n !(1+R)^{m} p_{v, m}(f) e^{\varphi_{v}(R)}}{R^{n}} .
\end{aligned}
$$

Using condition ( $\left.\mathrm{i}_{2}\right)$ on $\Phi$, we get

$$
(1+|x|)^{m}\left|f^{(n)}(x)\right| \leq e^{C(\nu, m)} n ! p_{v, m}(f) \frac{e^{\varphi_{v+1}(R)}}{R^{n}} .
$$

Further,

$$
\begin{aligned}
(1+|x|)^{m}\left|f^{(n)}(x)\right| & \leq e^{C(v, m)} n ! p_{v, m}(f) e^{\inf _{R \geq 1}\left(\varphi_{v+1}(R)-n \ln R\right)} \\
& =e^{C(v, m)} n ! p_{\nu, m}(f) e^{-\sup _{r \geq 0}\left(n r-\psi_{v+1}(R)\right)} \\
& =e^{C(v, m)} n ! p_{\nu, m}(f) e^{-\psi_{v+1}^{*}(n)}
\end{aligned}
$$

This means that

$$
\mathcal{R}_{m, v+1}\left(f_{\mid \mathbb{R}}\right) \leq e^{C(\nu, m)} p_{v, m}(f) .
$$

Therefore, $f_{\mid \mathbb{R}} \in \mathcal{E}\left(\psi_{v+1}^{*}\right)$. Thus, $f_{\mid \mathbb{R}} \in \mathcal{E}\left(\Psi^{*}\right)$. 
Proof of Theorem 1.2 First note that due to condition ( $\left.i_{3}\right)$ on $\Phi$ we have that for each $k \in \mathbb{N}$,

$$
\psi_{k}\left(t+\delta_{k}\right) \leq \psi_{k+1}(t)+\gamma_{k}, \quad t \geq 0
$$

where $\delta_{k}=\ln \sigma_{k}$. From this it easily follows that

$$
\psi_{k}^{*}(x)-\psi_{k+1}^{*}(x) \geq \delta_{k} x-\gamma_{k}, \quad x \geq 0 .
$$

Now let $f \in \mathcal{E}\left(\Psi^{*}\right)$. Then $f \in \mathcal{E}\left(\psi_{\nu}^{*}\right)$ for some $v \in \mathbb{N}$. Hence, for each $m \in \mathbb{Z}_{+}$we have

$$
(1+|x|)^{m}\left|f^{(n)}(x)\right| \leq \mathcal{R}_{m, v}(f) n ! e^{-\psi_{v}^{*}(n)}, \quad x \in \mathbb{R}, \quad n \in \mathbb{Z}_{+} .
$$

From this (taking into account that $\lim _{x \rightarrow+\infty} \psi_{v}^{*}(x) / x=+\infty$ ) we get that for each $\varepsilon>0$ there exists a constant $A_{\varepsilon}>0$ such that $\left|f^{(n)}(x)\right| \leq A_{\varepsilon} \varepsilon^{n} n$ ! for all $x \in \mathbb{R}$ and $n \in \mathbb{Z}_{+}$. So it is clear that the sequence $\left(\sum_{n=0}^{k} f^{(n)}(0) x^{n} / n !\right)_{k=0}^{\infty}$ converges to $f$ uniformly on compacts of the real axis and the series $\sum_{n=0}^{\infty} f^{(n)}(0) z^{n} / n$ ! converges uniformly on compacts of $\mathbb{C}$ and, hence, its sum $F_{f}(z)$ is an entire function. Obviously, $F_{f}(x)=f(x), x \in \mathbb{R}$. The uniqueness of holomorphic continuation is obvious.

Now we prove that $F_{f} \in E(\Phi)$. Let $m \in \mathbb{Z}_{+}$be arbitrary. Using the equality

$$
F_{f}(z)=\sum_{n=0}^{\infty} \frac{f^{(n)}(x)}{n !}(i y)^{n}, \quad z=x+i y, \quad x, y \in \mathbb{R}
$$

and inequality (4), we have

$$
\begin{aligned}
(1+|z|)^{m}\left|F_{f}(z)\right| & \leq \sum_{n=0}^{\infty} \frac{(1+|x|)^{m}(1+|y|)^{m+n}\left|f^{(n)}(x)\right|}{n !} \\
& \leq \mathcal{R}_{m, v}(f)(1+|y|)^{m} \sum_{n=0}^{\infty}(1+|y|)^{n} e^{-\psi_{v}^{*}(n)} \\
& =\mathcal{R}_{m, v}(f)(1+|y|)^{m} \sum_{n=0}^{\infty} \frac{(1+|y|)^{n}}{e^{\psi_{v+1}^{*}(n)}} e^{\psi_{v+1}^{*}(n)-\psi_{v}^{*}(n)}
\end{aligned}
$$

Now using inequality (3) and denoting $e^{\gamma_{v}+\delta_{v}} /\left(e^{\delta_{v}}-1\right)$ by $B_{v}$, we have

$$
\begin{aligned}
(1+|z|)^{m}\left|F_{f}(z)\right| & \leq B_{v} \mathcal{R}_{m, v}(f)(1+|y|)^{m} \sup _{n \in \mathbb{Z}_{+}} \frac{(1+|y|)^{n}}{e^{\psi_{v+1}^{*}(n)}} \\
& \leq B_{v} \mathcal{R}_{m, v}(f)(1+|y|)^{m} e^{\sup _{t \geq 0}\left(t \ln (1+|y|)-\psi_{v+1}^{*}(t)\right)}
\end{aligned}
$$

Thus,

$$
(1+|z|)^{m}\left|F_{f}(z)\right| \leq B_{v} \mathcal{R}_{m, v}(f) e^{\left(\psi_{v+1}^{*}\right)^{*}(\ln (1+|y|))+m \ln (1+|y|)}
$$


Recall that by condition ( $\mathrm{i}_{2}$ ) on $\Phi$ for each $k \in \mathbb{N}$ and $A>0$ there exists a constant $C(k, A)>0$ such that

$$
\varphi_{k}(x)+A \ln (1+x) \leq \varphi_{k+1}(x)+C(k, A), \quad x \geq 0 .
$$

Hence,

$$
\psi_{k}(x)+A x \leq \psi_{k+1}(x)+C(k, A), \quad x \geq 0 .
$$

From this we have that for all $\xi \geq 0$,

$$
\begin{aligned}
\psi_{k}^{*}(\xi) & =\sup _{x \geq 0}\left(\xi x-\psi_{k}(x)\right) \geq \sup _{x \geq 0}\left(\xi x-\psi_{k+1}(x)+A x\right)-C(k, A) \\
& =\sup _{x \geq 0}\left((\xi+A) x-\psi_{k+1}(x)\right)-C(k, A)=\psi_{k+1}^{*}(\xi+A)-C(k, A) .
\end{aligned}
$$

Then for all $x \geq 0$,

$$
\begin{aligned}
\left(\psi_{k}^{*}\right)^{*}(x) & =\sup _{x \geq 0}\left(x \xi-\psi_{k}^{*}(\xi)\right) \leq \sup _{x \geq 0}\left(x \xi-\psi_{k+1}^{*}(\xi+A)\right)+C(k, A) \\
& =\sup _{x \geq 0}\left(x(\xi+A)-\psi_{k+1}^{*}(\xi+A)\right)-A x+C(k, A) \\
& \leq\left(\psi_{k+1}^{*}\right)^{*}(x)-A x+C(k, A) .
\end{aligned}
$$

Thus, for each $k \in \mathbb{N}$ and $A>0$ we have

$$
\left(\psi_{k}^{*}\right)^{*}(x)+A x \leq\left(\psi_{k+1}^{*}\right)^{*}(x)+C(k, A), \quad x \geq 0 .
$$

Now with help of inequality (6) we obtain from estimate (5),

$$
(1+|z|)^{m}\left|F_{f}(z)\right| \leq B_{v} \mathcal{R}_{m, v}(f) e^{C(v+1, m)} e^{\left(\psi_{v+2}^{*}\right)^{*}(\ln (1+|y|))}
$$

It is clear that

$$
(1+|z|)^{m}\left|F_{f}(z)\right| \leq B_{\nu} \mathcal{R}_{m, v}(f) e^{C(v+1, m)} e^{\psi_{v+2}(\ln (1+|y|))} .
$$

Hence,

$$
(1+|z|)^{m}\left|F_{f}(z)\right| \leq B_{v} \mathcal{R}_{m, v}(f) e^{C(v+1, m)} e^{\varphi_{v+2}(1+|y|)}
$$

As functions of the family $\Phi$ are nondecreasing and satisfy condition ( $\left.\mathrm{i}_{3}\right)$, it is possible to find a constant $K_{v, m}>0$ such that for all $z \in \mathbb{C}$,

$$
(1+|z|)^{m}\left|F_{f}(z)\right| \leq K_{v, m} \mathcal{R}_{m, v}(f) e^{\varphi_{v+3}(|\operatorname{Im} z|)} .
$$

Thus, for each $m \in \mathbb{Z}_{+}, p_{v+3, m}\left(F_{f}\right) \leq K_{v, m} \mathcal{R}_{m, v}(f)$. Hence, $F_{f} \in E\left(\varphi_{v+3}\right)$. Thus, $F_{f} \in E(\Phi)$. 


\subsection{Additional information on a structure of the space $E(\Phi)$}

In Sect. 1.3 for each $v \in \mathbb{N}$ we introduced the space $\mathcal{H}\left(\varphi_{v}\right)$ as a projective limit of spaces

$$
\mathcal{H}_{k}\left(\varphi_{\nu}\right)=\left\{f \in H(\mathbb{C}): \mathcal{N}_{\nu, k}(f)=\sup _{z \in \mathbb{C}} \frac{|f(z)|(1+|z|)^{k}}{e^{\left(\psi_{\nu}^{*}\right)^{*}(\ln (1+|\operatorname{Im} z|))}}<\infty\right\}, \quad k \in \mathbb{Z}_{+},
$$

and then defined the space $\mathcal{H}(\Phi)$ as an inductive limit of spaces $\mathcal{H}\left(\varphi_{\nu}\right)$. Note that in view of inequality (6) the space $\mathcal{H}\left(\varphi_{\nu}\right)$ is continuously embedded in $\mathcal{H}\left(\varphi_{\nu+1}\right)$ for each $v \in \mathbb{N}$.

Remark that if the family $\Phi$ satisfies condition $\left(i_{3}\right)$ and functions $\psi_{m}$ are convex $[0, \infty)$, then $\mathcal{H}(\Phi)=E(\Phi)$. Indeed, for each $v \in \mathbb{N}$,

$$
\left(\psi_{v}^{*}\right)^{*}(\ln (1+t))=\psi_{v}(\ln (1+t))=\varphi_{v}(1+t) \geq \varphi_{v}(t), \quad t \geq 0
$$

On the other hand, for each $v \in \mathbb{N}$ there exists a constant $d_{v}>0$ such that $\varphi_{v}(1+t) \leq$ $\varphi_{v}\left(\sigma_{v} t\right)+d_{v}$ for all $t \geq 0$. Using this inequality and condition (i $\left.i_{3}\right)$, we have

$$
\left(\psi_{\nu}^{*}\right)^{*}(\ln (1+t)) \leq \psi_{\nu}(\ln (1+t))=\varphi_{\nu}(1+t) \leq \varphi_{\nu+1}(t)+\gamma_{\nu}+d_{\nu}, \quad t \geq 0 .
$$

From these inequalities our assertion easily follows. Using Theorems 1.1 and 1.2 we can prove a more strong assertion.

Proposition 3.1 Let the family $\Phi$ satisfy condition (i $\left.\mathrm{i}_{3}\right)$. Then $E(\Phi)=\mathcal{H}(\Phi)$.

Proof Let $v \in \mathbb{N}$ and $f \in \mathcal{H}\left(\varphi_{v}\right)$. Using nondecreasity of $\varphi_{v}$ and condition (i $\left.\mathrm{i}_{3}\right)$ on $\Phi$ we can find a constant $K_{v}>0$ such that for each $k \in \mathbb{Z}_{+}$,

$$
p_{\nu+1, k}(f) \leq K_{\nu} \mathcal{N}_{\nu, k}(f), \quad f \in \mathcal{H}\left(\varphi_{\nu}\right)
$$

From this it follows that the embedding $I: \mathcal{H}(\Phi) \rightarrow E(\Phi)$ is continuous.

The mapping $I$ is surjective too. Indeed, if $f \in E(\Phi)$ then $f \in E\left(\varphi_{\nu}\right)$ for some $v \in$ $\mathbb{N}$. Recall that by inequality (2) for each $k \in \mathbb{Z}_{+}$we have $\mathcal{R}_{k, v+1}\left(f_{\mid \mathbb{R}}\right) \leq e^{C_{v, k}} p_{v, k}(f)$. From this and inequality (7) (with $v$ replaced by $v+1$ ) we obtain

$$
\mathcal{N}_{v+3, k}(f) \leq A_{v, k} p_{v, k}(f),
$$

where $A_{k, v}$ is some positive number. Hence, $f \in \mathcal{H}\left(\varphi_{v+3}\right)$. Thus, $f \in \mathcal{H}(\Phi)$. Moreover, the last estimate shows that the inverse mapping $I^{-1}$ is continuous. Hence, the equality $E(\Phi)=\mathcal{H}(\Phi)$ is topological as well.

Remark 3.1 Let the family $\Phi$ satisfy conditions $\left(i_{1}\right)-\left(i_{3}\right)$. Consider the family $\widetilde{\Phi}$ consisting of functions $\left(\psi_{v}^{*}\right)^{*}(\ln (1+x))$. Obviously, for each $v \in \mathbb{N}$ functions $\left(\psi_{v}^{*}\right)^{*}(\ln (1+x))$ are continuous and nondecreasing on $[0, \infty)$. By inequality (6), for each $A>0$ we have

$$
\left(\psi_{v}^{*}\right)^{*}(\ln (1+x))+A \ln (1+x) \leq\left(\psi_{v+1}^{*}\right)^{*}(\ln (1+x))+C(v, A), \quad x \geq 0 .
$$


Since for each $v \in \mathbb{N}, \lim _{x \rightarrow+\infty}\left(\psi_{v}^{*}\right)^{*}(x) / e^{x}=+\infty$ (it follows from Lemma 2.1) then

$$
\lim _{x \rightarrow+\infty} \frac{\left(\psi_{v}^{*}\right)^{*}(\ln (1+x))}{x}=+\infty
$$

Thus, the family $\widetilde{\Phi}$ satisfies conditions of the form $\left(i_{1}\right)$ and $\left(i_{2}\right)$. Since $\Phi$ satisfies condition ( $\left.i_{3}\right)$, using inequality (3), it is easy to get

$$
\left(\psi_{v}^{*}\right)^{*}\left(t+\delta_{v}\right) \leq\left(\psi_{v+1}^{*}\right)^{*}(t)+\gamma_{k}, \quad t \geq 0
$$

Further, for all $x \geq 0$,

$$
\begin{aligned}
\left(\psi_{v}^{*}\right)^{*}\left(\ln \left(1+\sigma_{v} x\right)\right) & \leq\left(\psi_{v}^{*}\right)^{*}\left(\ln \sigma_{v}+\ln (1+x)\right) \\
& =\left(\psi_{v}^{*}\right)^{*}\left(\delta_{v}+\ln (1+x)\right) \leq\left(\psi_{v+1}^{*}\right)^{*}(\ln (1+x))+\gamma_{k} .
\end{aligned}
$$

Hence, the family $\widetilde{\Phi}$ satisfies a condition of the form $\left(i_{3}\right)$. Also note that functions $\left(\psi_{v}^{*}\right)^{*}(\ln (1+x))$ are convex on $[0, \infty)$. Hence, $\mathcal{H}(\widetilde{\Phi})=E(\widetilde{\Phi})$. By Proposition 3.1, we have that $E(\Phi)=\mathcal{H}(\Phi)$. Since $E(\widetilde{\Phi}) \equiv \mathcal{H}(\Phi)$, we finally obtain $E(\Phi)=$ $\mathcal{H}(\Phi)=\mathcal{H}(\widetilde{\Phi})$.

\section{Fourier transform of $E(\Phi)$}

First we will prove that under condition $\left(i_{3}\right)$ on $\Phi$ the space $G\left(\Psi^{*}\right)$ admits a more simple description. For $v \in \mathbb{N}$ and $m \in \mathbb{Z}_{+}$let

$$
Q_{m}\left(\psi_{v}^{*}\right)=\left\{f \in C^{m}\left(\mathbb{R}^{n}\right): N_{m, \psi_{v}^{*}}(f)=\max _{0 \leq n \leq m} \sup _{\substack{x \in \mathbb{R} \\ k \in \mathbb{Z}_{+}}} \frac{(1+|x|)^{k}\left|f^{(n)}(x)\right|}{k ! e^{-\psi_{v}^{*}(k)}}<\infty\right\}
$$

Let $Q\left(\psi_{v}^{*}\right)=\bigcap_{m \in \mathbb{Z}_{+}} Q_{m}\left(\psi_{v}^{*}\right), Q\left(\Psi^{*}\right)=\bigcup_{v=1}^{\infty} Q\left(\psi_{v}^{*}\right)$. With usual operations of addition and multiplication by complex numbers $Q\left(\psi_{v}^{*}\right)$ and $Q\left(\Psi^{*}\right)$ are linear spaces. The family of norms $N_{\psi_{m, v}^{*}}, m \in \mathbb{Z}_{+}$, defines a locally convex topology in $Q\left(\psi_{v}^{*}\right)$. Endow $Q\left(\Psi^{*}\right)$ with an inductive limit topology of spaces $Q\left(\psi_{v}^{*}\right)$.

Lemma 4.1 Let the family $\Phi$ satisfy condition $\left(\mathrm{i}_{3}\right)$. Then the spaces $Q\left(\Psi^{*}\right)$ and $G\left(\Psi^{*}\right)$ coincide.

Proof Let $v \in \mathbb{N}$ and $f \in Q\left(\psi_{v}^{*}\right)$. Then for each $m \in \mathbb{Z}_{+}, N_{m, \psi_{v}^{*}}(f)<\infty$. Since $\|f\|_{m, \psi_{v}^{*}} \leq N_{m, \psi_{v}^{*}}(f)$ for each $m \in \mathbb{Z}_{+}, f \in G_{m}\left(\psi_{v}^{*}\right)$. Thus, if $f \in Q\left(\Psi^{*}\right)$ then $f \in$ $G\left(\Psi^{*}\right)$ and, obviously, the embedding mapping $J: Q\left(\Psi^{*}\right) \rightarrow G\left(\Psi^{*}\right)$ is continuous.

Now let $v \in \mathbb{N}$ and $f \in G\left(\psi_{v}^{*}\right)$. Let $m \in \mathbb{Z}_{+}$be arbitrary and $n \in \mathbb{Z}_{+}$be such that $0 \leq n \leq m$. Then

$$
\left|f^{(n)}(x)\right| \leq\|f\|_{m, \psi_{v}^{*}} e^{-\psi_{v}^{*}(0)}, \quad x \in \mathbb{R} .
$$


Obviously,

$$
\sup _{\substack{|x| \leq 1 / \delta_{v} \\ k \in \mathbb{Z}_{+}}} \frac{(1+|x|)^{k}\left|f^{(n)}(x)\right|}{k ! e^{-\psi_{v+1}^{*}(k)}} \leq \sup _{\substack{|x| \leq 1 / \delta_{v} \\ k \in \mathbb{Z}_{+}}} \frac{\left(1+1 / \delta_{v}\right)^{k}\left|f^{(n)}(x)\right|}{k ! e^{-\psi_{v+1}^{*}(k)}}
$$

Now take into account that $\lim _{k \rightarrow \infty} e^{\psi_{v+1}^{*}(k)} /\left(k ! b^{k}\right)=0$ for each $b>0$, see Lemma 2.1. So we can find a constant $C_{v}>1$ such that

$$
\sup _{\substack{|x| \leq 1 / \delta_{v} \\ k \in \mathbb{Z}_{+}}} \frac{(1+|x|)^{k}\left|f^{(n)}(x)\right|}{k ! e^{-\psi_{v+1}^{*}(k)}} \leq C_{v} \sup _{|x| \leq 1 / \delta_{v}}\left|f^{(n)}(x)\right|
$$

Using (9), we get

$$
\sup _{\substack{|x| \leq 1 / \delta_{v} \\ k \in \mathbb{Z}_{+}}} \frac{(1+|x|)^{k}\left|f^{(n)}(x)\right|}{k ! e^{-\psi_{v+1}^{*}(k)}} \leq C_{v}\|f\|_{m, \psi_{v}^{*}} e^{-\psi_{v}^{*}(0)}
$$

Further,

$$
\begin{aligned}
\sup _{\substack{|x|>1 / \delta_{v} \\
k \in \mathbb{Z}_{+}}} \frac{(1+|x|)^{k}\left|f^{(n)}(x)\right|}{k ! e^{-\psi_{v+1}^{*}(k)}} & \leq \sup _{\substack{|x|>/ \delta_{v} \\
k \in \mathbb{Z}_{+}}} \frac{\left(1+\delta_{v}\right)^{k}|x|^{k}\left|f^{(n)}(x)\right|}{k ! e^{-\psi_{v+1}^{*}(k)}} \\
& =\sup _{\substack{|x|>1 / \delta_{v} \\
k \in \mathbb{Z}_{+}}} \frac{|x|^{k}\left|f^{(n)}(x)\right|}{k ! e^{-\psi_{v+1}^{*}(k)-k \ln \left(1+\delta_{v}\right)}} \\
& \leq \sup _{\substack{|x|>1 / \delta_{v} \\
k \in \mathbb{Z}_{+}}} \frac{|x|^{k}\left|f^{(n)}(x)\right|}{k ! e^{-\psi_{v+1}^{*}(k)-k \delta_{v}}}
\end{aligned}
$$

Using inequality (3), we have

$$
\sup _{\substack{|x|>1 / \delta_{v} \\ k \in \mathbb{Z}_{+}}} \frac{(1+|x|)^{k}\left|f^{(n)}(x)\right|}{k ! e^{-\psi_{v+1}^{*}(k)}} \leq e^{\gamma_{v}} \sup _{\substack{|x|>1 / \delta_{v} \\ k \in \mathbb{Z}_{+}}} \frac{|x|^{k}\left|f^{(n)}(x)\right|}{k ! e^{-\psi_{v}^{*}(k)}} \leq e^{\gamma_{v}}\|f\|_{m, \psi_{v}^{*}}
$$

From this and (10) we get for each $m \in \mathbb{Z}_{+}$,

$$
N_{m, \psi_{v+1}^{*}}(f) \leq M_{v}\|f\|_{m, \psi_{v}^{*}}, \quad f \in G\left(\psi_{v}^{*}\right)
$$

where $M_{v}=\max \left(C_{v} e^{-\psi_{v}^{*}(0)}, e^{\gamma_{v}}\right)$. Hence, $f \in Q\left(\psi_{v+1}^{*}\right)$. From (11) it follows that the inverse mapping $J^{-1}$ acts from $G\left(\Psi^{*}\right)$ to $Q\left(\Psi^{*}\right)$ and is continuous. Thus, the topological equality $Q\left(\psi^{*}\right)=G\left(\psi^{*}\right)$ is established. 
Proof of Theorem 1.3 Let $v \in \mathbb{N}$ and $f \in E\left(\varphi_{\nu}\right)$. Then for all $n \in \mathbb{Z}_{+}$and $x \in \mathbb{R}$ we have

$$
\left|\widehat{f}^{(n)}(x)\right| \leq \int_{\mathbb{R}} \frac{|f(\xi)|(1+|\xi|)^{n+2}}{1+\xi^{2}} d \xi \leq \pi p_{\nu, n+2}(f) e^{\varphi_{\nu}(0)} .
$$

Let $m \in \mathbb{N}, n \in \mathbb{Z}_{+}$and $x \in \mathbb{R}$. For arbitrary $\eta \in \mathbb{R}$,

$$
x^{m} \widehat{f}^{(n)}(x)=x^{m} \int_{\mathbb{R}} f(\zeta)(-i \zeta)^{n} e^{-i x \zeta} d \xi, \quad \zeta=\xi+i \eta, \quad \xi \in \mathbb{R} .
$$

From this we get

$$
\left|x^{m} \widehat{f}^{(n)}(x)\right| \leq \int_{\mathbb{R}}|f(\zeta)|(1+|\zeta|)^{n+2} e^{x \eta}|x|^{m} \frac{d \xi}{1+\xi^{2}} .
$$

If $x \neq 0$ then putting in the last inequality $\eta=-x t /|x|$ with $t>0$, we have

$$
\begin{aligned}
\left|x^{m} \widehat{f}^{(n)}(x)\right| & \leq \pi p_{v, n+2}(f) e^{-t|x|} e^{\varphi_{v}(t)}|x|^{m} \\
& \leq \pi p_{v, n+2}(f) e^{\sup _{r>0}(-t r+m \ln r)+\varphi_{v}(t)} \\
& \leq \pi p_{v, n+2}(f) e^{m \ln m-m-m \ln t+\varphi_{v}(t)}
\end{aligned}
$$

Since

$$
\begin{aligned}
& \inf _{t>0}(-m \ln t\left.+\varphi_{\nu}(t)\right)=-\sup _{t>0}\left(m \ln t-\varphi_{\nu}(t)\right) \\
& \leq-\sup _{t \geq 1}\left(m \ln t-\varphi_{\nu}(t)\right)=-\sup _{u \geq 0}\left(m u-\psi_{\nu}(u)\right)=-\psi_{\nu}^{*}(m)
\end{aligned}
$$

then from this and the previous estimate we obtain

$$
\left|x^{m} \widehat{f}^{(n)}(x)\right| \leq \pi p_{v, n+2}(f) e^{m \ln m-m-\psi_{v}^{*}(m)}, \quad x \neq 0
$$

If $x=0$ then $x^{m} \widehat{f}^{(n)}(x)=0$. From this and inequalities (12) and (13) (taking into account that $\left.\psi_{v}^{*}(0)=-\inf _{t \geq 0} \psi_{v}(t)=-\varphi_{\nu}(1) \leq-\varphi_{\nu}(0)\right)$ we have for all $m \in \mathbb{N}$ and $n \in \mathbb{Z}_{+}$,

$$
\left|x^{m} \widehat{f}^{(n)}(x)\right| \leq \pi p_{v, n+2}(f) m ! e^{-\psi_{v}^{*}(m)}, \quad x \in \mathbb{R} .
$$

Thus, for all $k \in \mathbb{Z}_{+}$we get

$$
\max _{0 \leq n \leq k} \sup _{\substack{x \in \mathbb{R} \\ m \in \mathbb{Z}_{+}}} \frac{\left|x^{m} \widehat{f}^{(n)}(x)\right|}{m ! e^{-\psi_{v}^{*}(m)}} \leq \pi p_{v, k+2}(f), \quad f \in E\left(\varphi_{v}\right) .
$$

In other words, $\|\widehat{f}\|_{k, \psi_{v}^{*}} \leq \pi p_{v, k+2}(f), f \in E\left(\varphi_{\nu}\right)$. From this inequality it follows that the linear mapping $\mathcal{F}: f \in E(\Phi) \rightarrow \widehat{f}$ acts from $E(\Phi)$ to $G\left(\Psi^{*}\right)$ and is continuous. 
Let us show that $\mathcal{F}$ is surjective. Let $g \in G\left(\Psi^{*}\right)$, then $g \in G\left(\psi_{v}^{*}\right)$ for some $v \in \mathbb{N}$. By the proof of Lemma 4.1, $g \in Q\left(\psi_{v+1}^{*}\right)$. Hence, for all $m, k \in \mathbb{Z}_{+}$,

$$
(1+|x|)^{k}\left|g^{(j)}(x)\right| \leq N_{m, \psi_{v+1}^{*}}(g) k ! e^{-\psi_{v+1}^{*}(k)}, \quad x \in \mathbb{R}, \quad j=0, \ldots, m
$$

Put

$$
f(\xi)=\frac{1}{2 \pi} \int_{\mathbb{R}} g(x) e^{i x \xi} d x, \quad \xi \in \mathbb{R}
$$

and show that $f$ satisfies assumptions of Theorem 1.2. Let $m, n \in \mathbb{Z}_{+}$be arbitrary, $r=\min (m, n)$ and $\xi \in \mathbb{R}$. Then

$$
\begin{aligned}
(i \xi)^{m} f^{(n)}(\xi) & =\frac{1}{2 \pi} \int_{\mathbb{R}}\left(g(x)(i x)^{n}\right)^{(m)} e^{i x \xi} d x \\
& =\frac{(-1)^{m}}{2 \pi} \int_{\mathbb{R}} \sum_{j=0}^{r} C_{m}^{j} g^{(m-j)}(x)\left((i x)^{n}\right)^{(j)} e^{i x \xi} d x .
\end{aligned}
$$

From this we have

$$
\begin{aligned}
\left|\xi^{m} f^{(n)}(\xi)\right| & \leq \frac{1}{2 \pi} \sum_{j=0}^{r} C_{m}^{j} \int_{\mathbb{R}}\left|g^{(m-j)}(x)\right| \frac{n !}{(n-j) !}|x|^{n-j} d x \\
& \leq \frac{1}{2 \pi} \sum_{j=0}^{r} C_{m}^{j} \frac{n !}{(n-j) !} \int_{\mathbb{R}}\left|g^{(m-j)}(x)\right|(1+|x|)^{n-j+2} \frac{d x}{1+x^{2}} \\
& \leq \frac{1}{2} \sum_{j=0}^{r} C_{m}^{j} \frac{n !}{(n-j) !} N_{m, \psi_{v+1}^{*}}(g)(n-j+2) ! e^{-\psi_{v+1}^{*}(n-j+2)} .
\end{aligned}
$$

Continuing the estimate, we get

$$
\left|\xi^{m} f^{(n)}(\xi)\right| \leq \frac{(n+2) ! m !}{2} N_{m, \psi_{v+1}^{*}}(g) \sum_{j=0}^{r} \frac{e^{-\psi_{v+1}^{*}(n-j)}}{j !} .
$$

Recall now that by assumption of theorem, for each $s \in \mathbb{N}$ there exist numbers $\lambda_{s}>1$, $h_{s} \in\left(1, \sigma_{s+1}\right)$ and $l_{s}>0$ such that

$$
\lambda_{s} \varphi_{s}(x) \leq \varphi_{s+1}\left(h_{s} x\right)+l_{s}, \quad x \geq 0 .
$$

From this it follows that

$$
\lambda_{s} \psi_{s}(x) \leq \psi_{s+1}\left(x+\tau_{s}\right)+l_{s}, \quad x \geq 0,
$$


where $\tau_{s}=\ln h_{s}$. Then, by Lemma 2.2, there exists a constant $K_{s}>0$ such that for all nonnegative numbers $\alpha$ and $\beta$ satisfying the equality $\alpha+\beta=\lambda_{s}$ we have

$$
\psi_{s+1}^{*}(\alpha x+\beta y) \leq \alpha \psi_{s}^{*}(x)+\beta \psi_{s}^{*}(y)+\tau_{s}(\alpha x+\beta y)+K_{s}, \quad x, y \geq 0 .
$$

Using (15), we have from (14)

$$
\begin{array}{r}
\left|\xi^{m} f^{(n)}(\xi)\right| \leq b_{v, 1}(n+2) ! N_{m, \psi_{v+1}^{*}}(g) m ! e^{-\psi_{v+2}^{*}(n)+\tau_{v+1} n} \\
\sum_{j=0}^{r} \frac{e^{\left(\lambda_{v+1}-1\right) \psi_{v+1}^{*}\left(j /\left(\lambda_{v+1}-1\right)\right)}}{j !},
\end{array}
$$

where $b_{v, 1}=e^{K_{v+1}} / 2$. Obviously, there exists a constant $b_{v, 2}>0$ such that for all $m, n \in \mathbb{Z}_{+}$,

$$
\left|\xi^{m} f^{(n)}(\xi)\right| \leq b_{v, 2} n ! N_{m, \psi_{v+1}^{*}}(g) m ! e^{-\psi_{v+2}^{*}(n)+\delta_{v+2} n} \sum_{j=0}^{\infty} \frac{e^{\left(\left(\lambda_{v+1}-1\right) \psi_{v+1}\right)^{*}(j)}}{j !}
$$

Note that the series $\sum_{j=0}^{\infty} e^{\left(\left(\lambda_{v+1}-1\right) \psi_{\nu+1}\right)^{*}(j)} / j$ ! converges, see Corollary 2.1. Now using inequality (3), we get

$$
\left|\xi^{m} f^{(n)}(\xi)\right| \leq b_{v, 2} n ! N_{m, \psi_{v+1}^{*}}(g) m ! e^{-\psi_{v+3}^{*}(n)}
$$

where $b_{v, 3}=b_{v, 2} e^{\gamma_{v+2}} \sum_{j=0}^{\infty} e^{\left(\left(\lambda_{v+1}-1\right) \psi_{v+1}\right)^{*}(j)} / j$ !. From this we have that for each $m \in \mathbb{Z}_{+}$and all $n \in \mathbb{Z}_{+}, \xi \in \mathbb{R}$,

$$
(1+|\xi|)^{m}\left|f^{(n)}(\xi)\right| \leq 2^{m} b_{v, 3} n ! N_{m, \psi_{v+1}^{*}}(g) m ! e^{-\psi_{v+3}^{*}(n)}
$$

By Theorem 1.2, $f$ can be holomorphically continued (uniquely) to an entire function $F_{f}$ belonging to $E(\Phi)$. Obviously, $g=\mathcal{F}\left(F_{f}\right)$. The proof of Theorem 1.2 (in particular, inequalities (4) and (8)) indicates that there is a constant $K(v, m)>0$ such that for $z \in \mathbb{C}, z=x+i y$,

$$
(1+|z|)^{m}\left|F_{f}(z)\right| \leq K(v, m) N_{m, \psi_{v+1}^{*}}(g) e^{\varphi_{v+6}(|y|)} .
$$

Thus, for each $m \in \mathbb{Z}_{+}$,

$$
p_{\nu+6, m}\left(\mathcal{F}^{-1} g\right) \leq K(\nu, m) N_{m, \psi_{v+1}^{*}}(g), \quad g \in G\left(\psi_{v}^{*}\right)
$$

Taking into account inequality (11), we get

$$
p_{\nu+6, m}\left(\mathcal{F}^{-1} g\right) \leq M_{\nu} K(\nu, m)\|g\|_{m, \psi_{v}^{*}}, \quad g \in G\left(\psi_{\nu}^{*}\right) .
$$

From this estimate it follows that the inverse mapping $\mathcal{F}^{-1}$ is continuous. 
Thus, it is proved that Fourier transform establishes a topological isomorphism between spaces $E(\Phi)$ and $G\left(\Psi^{*}\right)$.

\section{Special case of a family $\Phi$}

In the proof of Theorem 1.4 the following three lemmas will be used.

Lemma 5.1 Let $g$ be a real-valued continuous function on $[0, \infty)$ such that $\lim _{x \rightarrow+\infty} g(x) / x=+\infty$. Then for each $\delta>0$,

$$
\lim _{x \rightarrow+\infty} \frac{g^{*}((1+\delta) x)-g^{*}(x)}{x}=+\infty .
$$

Proof Let $\delta>0$ be arbitrary. For each $x>0$ denote by $\xi(x)$ a point where the supremum of the function $u_{x}(\xi)=x \xi-g(\xi)$ over $[0, \infty)$ is attained. Note that $\xi(x) \rightarrow+\infty$ as $x \rightarrow+\infty$. Otherwise there are a number $M>0$ and a sequence $\left(x_{j}\right)_{j=1}^{\infty}$ of positive numbers $x_{j}$ converging to $+\infty$ such that $\xi\left(x_{j}\right) \leq M$. Then $g^{*}\left(x_{j}\right)$ $=x_{j} \xi\left(x_{j}\right)-g\left(\xi\left(x_{j}\right)\right)$. But it contradicts to the fact that $\lim _{x \rightarrow+\infty} g^{*}(x) / x=+\infty$. Thus, $\lim _{x \rightarrow+\infty} \xi(x)=+\infty$. From this and the inequality

$$
g^{*}((1+\delta) x)-g^{*}(x) \geq(1+\delta) x \xi(x)-g(\xi(x))-x \xi(x)+g(\xi(x))=\delta x \xi(x),
$$

for all $x>0$, the assertion of lemma follows.

The proof of the following lemma essentially rests on ideas of Napalkov and Popenov from [7, Lemma 4].

Lemma 5.2 Let $u \in V$, then there exists a constant $K>0$ depending on $u$ such that

$$
(u[e])^{*}(t)+\left(u^{*}[e]\right)^{*}(t) \geq t \ln t-t-K, \quad t>0 .
$$

Proof It is known [4] that there exists a function $v \in V_{u}$ such that

$$
\lim _{t \rightarrow+\infty} \frac{v(t)}{t}=\lim _{t \rightarrow 0+} \frac{t}{v(t)}=+\infty
$$

Since $|v(t)-u(t)| \leq A, t \geq 0$, for some $A>0$ then

$$
\begin{aligned}
& \left|(v[e])^{*}(x)-(u[e])^{*}(x)\right| \leq A, \quad x \geq 0, \\
& \left|\left(v^{*}[e]\right)^{*}(x)-\left(u^{*}[e]\right)^{*}(x)\right| \leq A, \quad x \geq 0 \text {. }
\end{aligned}
$$

Let $t>0$ be arbitrary. Note that

$$
\begin{gathered}
\sup _{\xi \geq 0}\left(t \xi-v\left(e^{\xi}\right)\right) \geq \sup _{\xi \in \mathbb{R}}\left(t \xi-v\left(e^{\xi}\right)\right)-v(1), \\
\sup _{\mu \geq 0}\left(t \mu-v^{*}\left(e^{\mu}\right)\right) \geq \sup _{\mu \in \mathbb{R}}\left(t \mu-v^{*}\left(e^{\mu}\right)\right)-v^{*}(1) .
\end{gathered}
$$


Hence, for each $\xi, \mu \in \mathbb{R}$,

$$
(v[e])^{*}(t)+\left(v^{*}[e]\right)^{*}(t) \geq t(\xi+\mu)-\left(v\left(e^{\xi}\right)+v^{*}\left(e^{\mu}\right)\right)-v(1)-v^{*}(1) .
$$

Using simple geometrical ideas, it is not difficult to see (firstly it was noticed by Popenov) that there exists a unique point $x_{0}>0$ such that

$$
u_{-}^{\prime}\left(x_{0}\right) \leq \frac{t}{x_{0}} \leq u_{+}^{\prime}\left(x_{0}\right)
$$

(here $u_{-}^{\prime}\left(x_{0}\right)$ is the left derivative at $x_{0}$ and $u_{+}^{\prime}\left(x_{0}\right)$ is the right derivative at $\left.x_{0}\right)$ and if $x_{0}^{*}=t / x_{0}$ then the following equality holds:

$$
u\left(x_{0}\right)+u^{*}\left(x_{0}^{*}\right)=t
$$

Putting in (17), $\xi=\ln x_{0}, \mu=\ln x_{0}^{*}$, we get

$$
(v[e])^{*}(t)+\left(v^{*}[e]\right)^{*}(t) \geq t \ln t-t-v(1)-v^{*}(1), \quad t>0 .
$$

Using inequalities (16), we get from (18)

$$
(u[e])^{*}(t)+\left(u^{*}[e]\right)^{*}(t) \geq t \ln t-t-v(1)-v^{*}(1)-2 A, \quad t>0 .
$$

It remains to put $K=v(1)+v^{*}(1)+2 A$.

The following lemma was proved in $[5,6]$.

Lemma 5.3 Let a lower semi-continuous function $u:[0, \infty) \rightarrow \mathbb{R}$ be such that $\lim _{x \rightarrow+\infty} u(x) / x=+\infty$. Then

$$
(u[e])^{*}(x)+\left(u^{*}[e]\right)^{*}(x) \leq x \ln x-x, \quad x>0 .
$$

Proof of Theorem 1.4 First note that using convexity of functions of the family $\Phi$ and condition (i3) on $\Phi$ we easily obtain that for each $k \in \mathbb{N}$,

$$
\sigma_{k} \varphi_{k}(x) \leq \varphi_{k+1}(x)+\gamma_{k}+\left(\sigma_{k}-1\right) \varphi_{k}(0), \quad x \geq 0 .
$$

In particular, this means that condition $\left(\mathrm{i}_{4}\right)$ holds in our case trivially with $\lambda_{k}=\sigma_{k}$ and $l_{k}=\gamma_{k}+\left(\sigma_{k}-1\right) \varphi_{k}(0)$. Also from the last inequality it follows that for each $k \in \mathbb{N}$ we have

$$
\sigma_{k} \psi_{k}(x) \leq \psi_{k+1}(x)+\gamma_{k}+\left(\sigma_{k}-1\right) \varphi_{k}(0), \quad x \geq 0
$$

Hence, by Lemma 2.2, for each $k \in \mathbb{N}$ there exists a number $A_{k}>0$ such that for all nonnegative numbers $\alpha$ and $\beta$ satisfying the equality $\alpha+\beta=\sigma_{k}$ we have

$$
\psi_{k+1}^{*}(\alpha x+\beta y) \leq \alpha \psi_{k}^{*}(x)+\beta \psi_{k}^{*}(y)+A_{k}, \quad x, y \geq 0 .
$$


Now let $v \in \mathbb{N}$ and $f \in G\left(\psi_{v}^{*}\right)$. Fix $m \in \mathbb{Z}_{+}$. Since $f \in Q\left(\psi_{v+1}^{*}\right)$ (see the proof of Lemma 4.1), for all $k \in \mathbb{Z}_{+}$and $n \in \mathbb{Z}_{+}$such that $0 \leq n \leq m$,

$$
\left|f^{(n)}(x)\right| \leq \frac{N_{m, \psi_{v+1}^{*}}(f) k ! e^{-\psi_{v+1}^{*}(k)}}{(1+|x|)^{k}}, \quad x \in \mathbb{R} .
$$

Using inequality (19), nondecreasity of $\psi_{v+1}^{*}$ and taking into account that $j !<$ $3 j^{j+1} / e^{j}$ for all $j \in \mathbb{N}$, we have that for all $k \in \mathbb{N}, t \in[k, k+1)$ and $\mu \geq 1$,

$$
\begin{aligned}
\frac{k ! e^{-\psi_{v+1}^{*}(k)}}{\mu^{k}} & \leq \frac{3 k^{k+1} e^{-\psi_{v+1}^{*}(k)}}{e^{k} \mu^{k}} \\
& \leq \frac{3 \mu t^{t+1} e^{-\psi_{v+2}^{*}(t)+\left(\sigma_{v+1}-1\right) \psi_{v+1}^{*}\left(1 /\left(\sigma_{v+1}-1\right)\right)+A_{v+1}+1}}{e^{t} \mu^{t}}
\end{aligned}
$$

From this using inequality (3), we obtain

$$
\frac{k ! e^{-\psi_{v+1}^{*}(k)}}{\mu^{k}} \leq C_{v, 1} \mu e^{(t+1) \ln t-\psi_{v+3}^{*}(t)-t \ln e \mu-\delta_{v+2} t},
$$

where $C_{v, 1}=3 e^{\left(\sigma_{v+1}-1\right) \psi_{v+1}^{*}\left(1 /\left(\sigma_{v+1}-1\right)\right)+A_{v+1}+\gamma_{v+2}+1}$. Using Lemma 5.2, we get for some $C_{v, 2}>0$ (not depending on $k$ and $t$ ),

$$
\frac{k ! e^{-\psi_{v+1}^{*}(k)}}{\mu^{k}} \leq C_{\nu, 2} \mu e^{\left(\varphi_{\nu+3}^{*}[e]\right)^{*}(t)-t \ln \mu+\ln t-\delta_{v+2} t} .
$$

We can choose a constant $C_{v, 3}>0$ (not depending on $k$ and $t$ ) so that

$$
\frac{k ! e^{-\psi_{v+1}^{*}(k)}}{\mu^{k}} \leq C_{v, 3} \mu e^{\left(\varphi_{\nu+3}^{*}[e]\right)^{*}(t)-t \ln \mu} .
$$

From this it follows that

$$
\inf _{k \in \mathbb{N}} \frac{k ! e^{-\psi_{v+1}^{*}(k)}}{\mu^{k}} \leq C_{v, 3} \mu e^{\inf _{t \geq 1}\left(\left(\varphi_{v+3}^{*}[e]\right)^{*}(t)-t \ln \mu\right)}
$$

Obviously,

$$
\begin{array}{r}
\inf _{t \geq 1}\left(\left(\varphi_{\nu+3}^{*}[e]\right)^{*}(t)-t \ln \mu\right) \leq-\ln \mu+\left(\varphi_{\nu+3}^{*}[e]\right)^{*}(1), \\
\inf _{0<t \leq 1}\left(\left(\varphi_{\nu+3}^{*}[e]\right)^{*}(t)-t \ln \mu\right) \geq-\ln \mu+\left(\varphi_{\nu+3}^{*}[e]\right)^{*}(0) .
\end{array}
$$

Consequently,

$$
\begin{aligned}
\inf _{t \geq 1}\left(\left(\varphi_{\nu+3}^{*}[e]\right)^{*}(t)-t \ln \mu\right) \leq \inf _{0<t \leq 1} & \left(\left(\varphi_{\nu+3}^{*}[e]\right)^{*}(t)-t \ln \mu\right) \\
& +\left(\varphi_{\nu+3}^{*}[e]\right)^{*}(1)-\left(\varphi_{\nu+3}^{*}[e]\right)^{*}(0)
\end{aligned}
$$


Denoting $\left(\varphi_{v+3}^{*}[e]\right)^{*}(1)-\left(\varphi_{v+3}^{*}[e]\right)^{*}(0)$ by $m_{v}$, we have

$$
\inf _{t \geq 1}\left(\left(\varphi_{\nu+3}^{*}[e]\right)^{*}(t)-t \ln \mu\right) \leq \inf _{t>0}\left(\left(\varphi_{\nu+3}^{*}[e]\right)^{*}(t)-t \ln \mu\right)+m_{v}
$$

With help of this inequality we have from (21)

$$
\inf _{k \in \mathbb{N}} \frac{k ! e^{-\psi_{v+1}^{*}(k)}}{\mu^{k}} \leq C_{v, 3} e^{m_{v}} \mu e^{\inf _{t>0}\left(\left(\varphi_{v+3}^{*}[e]\right)^{*}(t)-t \ln \mu\right)} .
$$

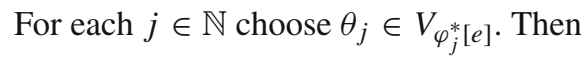

$$
\begin{aligned}
\left|\theta_{j}(\xi)-\varphi_{j}^{*}[e](\xi)\right| & \leq r_{j}, & & \xi \geq 0, \\
\left|\theta_{j}^{*}(\xi)-\left(\varphi_{j}^{*}[e]\right)^{*}(\xi)\right| & \leq r_{j}, & & \xi \geq 0,
\end{aligned}
$$

where $r_{j}$ is some positive number depending on $\varphi_{j}^{*}[e]$ and $\theta_{j}$. From (22) using inequality (24), we have

$$
\inf _{k \in \mathbb{N}} \frac{k ! e^{-\psi_{v+1}^{*}(k)}}{\mu^{k}} \leq C_{\nu, 4} \mu e^{\inf _{t>0}\left(\theta_{v+3}^{*}(t)-t \ln \mu\right)},
$$

where $C_{v, 4}=C_{v, 3} e^{m_{v}+r_{v+3}}$. Using the Young inversion formula, we obtain

$$
\inf _{k \in \mathbb{N}} \frac{k ! e^{-\psi_{v+1}^{*}(k)}}{\mu^{k}} \leq C_{v, 4} \mu e^{-\theta_{v+3}(\ln \mu)}
$$

From this, using inequality (23), we have

$$
\inf _{k \in \mathbb{N}} \frac{k ! e^{-\psi_{v+1}^{*}(k)}}{\mu^{k}} \leq C_{v, 5} \mu e^{-\varphi_{v+3}^{*}[e](\ln \mu)},
$$

where $C_{\nu, 5}=C_{v, 4} e^{r_{v+3}}$. In other words,

$$
\inf _{k \in \mathbb{N}} \frac{k ! e^{-\psi_{v+1}^{*}(k)}}{\mu^{k}} \leq C_{v, 5} \mu e^{-\varphi_{v+3}^{*}(\mu)}
$$

Using this inequality and nondecreasity of $\varphi_{\nu+3}^{*}$, we have

$$
\inf _{k \in \mathbb{N}} \frac{k ! e^{-\psi_{v+1}^{*}(k)}}{(1+|x|)^{k}} \leq C_{v, 5}(1+|x|) e^{-\varphi_{v+3}^{*}(|x|)}, \quad x \in \mathbb{R} .
$$

Note that using condition $\left(\mathrm{i}_{3}\right)$ on $\Phi$, it is easy to obtain that for each $j \in \mathbb{N}$,

$$
\varphi_{j+1}^{*}(\xi) \leq \varphi_{j}^{*}\left(\frac{\xi}{\sigma_{j}}\right)+\gamma_{j}, \quad \xi \geq 0
$$


Hence,

$$
\varphi_{j}^{*}(\xi)-\varphi_{j+1}^{*}(\xi) \geq \varphi_{j}^{*}(\xi)-\varphi_{j}^{*}\left(\frac{\xi}{\sigma_{j}}\right)-\gamma_{j}, \quad \xi \geq 0 .
$$

From this and Lemma 5.1 we get

$$
\lim _{\xi \rightarrow+\infty} \frac{\varphi_{j}^{*}(\xi)-\varphi_{j+1}^{*}(\xi)}{\xi}=+\infty
$$

Going back to (25), we obtain with help of (27)

$$
\inf _{k \in \mathbb{N}} \frac{k ! e^{-\psi_{v+1}^{*}(k)}}{(1+|x|)^{k}} \leq C_{v, 6} e^{-\varphi_{v+4}^{*}(|x|)}, \quad x \in \mathbb{R},
$$

where $C_{v, 6}$ is some positive number. From this and inequality (20), we obtain that for $n \in \mathbb{Z}_{+}$such that $0 \leq n \leq m$,

$$
\left|f^{(n)}(x)\right| \leq C_{v, 6} N_{m, \psi_{v+1}^{*}}(f) e^{-\varphi_{v+4}^{*}(|x|)}, \quad x \in \mathbb{R} .
$$

This means that

$$
q_{m, v+4}(f) \leq C_{v, 6} N_{m, \psi_{v+1}^{*}}(f), \quad f \in G\left(\psi_{v}^{*}\right) .
$$

Taking into account inequality (11), we have

$$
q_{m, v+4}(f) \leq C_{\nu, 7}\|f\|_{m, \psi_{v}^{*}}, \quad f \in G\left(\psi_{v}^{*}\right)
$$

where $C_{v, 7}$ is some positive constant depending on $v$. From this it follows that the identity mapping $T$ acts from $G\left(\Psi^{*}\right)$ to $G S\left(\Phi^{*}\right)$ continuously.

Show that $T$ is surjective. Let $f \in G S\left(\Phi^{*}\right)$. Then $f \in G S\left(\varphi_{v}^{*}\right)$ for some $v \in \mathbb{N}$. Let $m \in \mathbb{Z}_{+}$be fixed, $x$ be arbitrary. For all $n \in \mathbb{Z}_{+}$such that $0 \leq n \leq m$ we have

$$
\left|f^{(n)}(x)\right| \leq q_{m, v}(f) e^{-\varphi_{v}^{*}(|x|)}
$$

Using inequality (26), we have from (28)

$$
\left|f^{(n)}(x)\right| \leq e^{\gamma_{v}} q_{m, v}(f) e^{-\varphi_{v+1}^{*}\left(\sigma_{v}|x|\right)}
$$

Obviously, there exists a constant $\alpha_{v}>1$ (not depending on $x$ and $n$ ) such that

$$
\left|f^{(n)}(x)\right| \leq \alpha_{\nu} q_{m, v}(f) e^{-\varphi_{v+1}^{*}(|x|+1)}
$$

In other words,

$$
\left|f^{(n)}(x)\right| \leq \alpha_{\nu} q_{m, v}(f) e^{-\varphi_{v+1}^{*}[e](\ln (|x|+1))}
$$


From this we have

$$
\left|f^{(n)}(x)\right| \leq \alpha_{v} q_{m, v}(f) e^{-\sup _{t>0}\left(t \ln (|x|+1)-\left(\varphi_{v+1}^{*}[e]\right)^{*}(t)\right)}
$$

Now using Lemma 5.3, we get

$$
\left|f^{(n)}(x)\right| \leq \alpha_{\nu} q_{m, v}(f) e^{-\sup _{t>0}\left(t \ln (e(|x|+1))-t \ln t+\psi_{v+1}^{*}(t)\right)} .
$$

Consequently, for all $k \in \mathbb{N}$,

$$
\left|f^{(n)}(x)\right| \leq \alpha_{\nu} q_{m, v}(f) \frac{k^{k} e^{-\psi_{v+1}^{*}(k)}}{(e(1+|x|))^{k}} .
$$

From this and (28) it follows that

$$
(1+|x|)^{k}\left|f^{(n)}(x)\right| \leq \alpha_{\nu} q_{m, v}(f) k ! e^{-\psi_{v+1}^{*}(k)}, \quad k \in \mathbb{Z}_{+} .
$$

This means that

$$
\|f\|_{m, \psi_{v+1}^{*}} \leq \alpha_{\nu} q_{m, v}(f) .
$$

Since $m \in \mathbb{Z}_{+}$was arbitrary, $f \in G\left(\psi_{v+1}^{*}\right)$. Hence, $f \in G\left(\Psi^{*}\right)$.

From (29) it follows that the mapping $T^{-1}$ is continuous. Thus, the spaces $G\left(\Psi^{*}\right)$ and $G S\left(\Phi^{*}\right)$ coincide.

Acknowledgments The authors are very grateful to the referee for careful reading, valuable comments and suggestions.

\section{References}

1. Gelfand, I.M., Shilov, G.E.: Generalized Functions, vols. 2-3. Academic Press, New York $(1968,1967)$

2. Gurevich, B.L.: New types of spaces of fundamental and generalized functions and Cauchy's problem for systems of finite difference equations. Dokl. Akad. Nauk SSSR (N.S.) 99(6), 893-895 (1954) (in Russian)

3. Gurevich, B.L.: New Types of Spaces of Fundamental and Generalized Functions and the Cauchy Problem for Operator Equations. Dissertation, Kharkov (1956) (in Russian)

4. Krasnosel'skii, M.A., Rutickii, Ya.B.: Convex Functions and Orlicz Spaces. P. Noordhoff, Groningen (1961)

5. Musin, I.Kh.: Approximation by polynomials in a weighted space of infinitely differentiable functions with an application to hypercyclicity. Extracta Math. 27(1), 75-90 (2012)

6. Musin, I.Kh., Popenov, S.V.: On a weighted space of infinitely differentiable functions in $\mathbb{R}^{n}$. Ufa Math. J. 2(3), 52-60 (2010) (in Russian)

7. Napalkov, V.V., Popenov, S.V.: On the Laplace transform of functionals in a weighted Bergman space of entire functions in $\mathbb{C}^{n}$. Dokl. Akad. Nauk 352(5), 595-597 (1997) (in Russian)

8. Sedletskiǔ, A.M.: Classes of entire functions that rapidly decrease on the real axis. Moscow Univ. Math. Bull. 60(1), 37-43 (2005)

9. Sedletskiı̌, A.M.: Classes of entire functions that decrease rapidly on the real axis: theory and applications. Sb. Math. 199(1-2), 131-157 (2008) 